\title{
On Positive-Realness and Lyapunov Functions for Switched Linear Differential Systems
}

\author{
J. C. Mayo-Maldonado and P. Rapisarda
}

\begin{abstract}
We show new results about Lyapunov stability of switched linear differential systems (SLDS) using the concept of positive realness. The main results include stability conditions for a class of SLDS with augmented banks and the parametrization of families of asymptotically stable SLDS with three modes. Such conditions can be verified using LMIs that can be directly set up from the higher-order differential equations describing the modes.
\end{abstract}

Index Terms-Positive-realness, quadratic differential forms, stability, switched systems.

\section{INTRODUCTION}

Usually switched systems are studied using state space-(see e.g., [2], [6]) or descriptor form-(see [18]) representations, together with a switching rule that determines the activation of modes; furthermore, state reset maps can be incorporated to act at the switching times. In such classic approaches, the dynamical modes share a global state space. However, we argued in [10] that there are many switched systems for which no compelling reason exists to use a global state space. In [10], it is also shown that the use of state space representations themselves is not necessary, and that switched systems can be studied directly in higher-order terms, i.e., using sets of linear differential equations to describe the modes.

Posive-realness has played an important role in the study of switched systems. For instance it is well-known that if an open-loop transfer function of a system is positive-real, then all stable closedloop systems obtained from it by state feedback share a common quadratic Lyapunov function (see Sec. 2.3.2 of [6] and [16], [17]). Other contributions regarding quadratic stability of a class of switched linear systems are shown in [7] and [15]. A contribution in stability of switched systems in descriptor form, using an extended version of the positive-real lemma, can be found in [20]. Recently, positiverealness has also played a role in dimensionality reduction for switching descriptor systems, see [13], [14]. In this note, we provide new results generalising material included in [8] for the special case of scalar SLDS and the results in [10, Sec. IV] regarding the role of positive-realness in stability of a class of SLDS. We show sufficient conditions for stability for pairs of multivariable dynamical modes that do not share the same state space using positive-real completions. The conditions are based on LMIs that can be directly set-up from the higher-order differential equations describing the modes. Moreover, we show that the existence of positive-real completions imply also the existence of additional dynamical modes in extended banks whose

Manuscript received March 6, 2015; revised September 12, 2015; accepted September 21, 2015. Date of publication September 30, 2015; date of current version July 22, 2016. Recommended by Associate Editor H. L. Trentelman.

J. C. Mayo-Maldonado is with the School of Engineering and Sciences, Tecnologico de Monterrey, 64849 Campus Monterrey, Mexico (e-mail: jcmayo@ itesm.mx).

P. Rapisarda is with the VLC group, School of Electronics and Computer Science, University of Southampton, Southampton SO17 1BJ, U.K. (e-mail: pr3@ecs.soton.ac.uk).

Digital Object Identifier 10.1109/TAC.2015.2484329 stability conditions are analogous with those of the original bank. Finally, stability of parameter depending families of SLDS with three dynamical modes is also proved.

We use the following notation. The space of real vectors with $\mathrm{n}$ components is denoted by $\mathbb{R}^{\mathrm{n}}$. $\mathfrak{C}^{\infty}\left(\mathbb{R}, \mathbb{R}^{\mathrm{w}}\right)$ represents the set of infinitely-differentiable functions from $\mathbb{R}$ to $\mathbb{R}^{\mathrm{w}}$. The space of $m \times n$ real matrices by $\mathbb{R}^{\mathrm{m} \times \mathrm{n}}$. The space of real matrices with $\mathrm{n}$ columns and a finite unspecified number of rows is denoted by $\mathbb{R}^{\bullet \times n}$. The ring of polynomials with real coefficients in $\xi$ is denoted by $\mathbb{R}[\xi]$. $\mathbb{R}^{\mathrm{m} \times \mathrm{n}}[\xi]$ is the space of $\mathrm{m} \times \mathrm{n}$ polynomial matrices in $\xi$. The space of $\mathrm{m} \times \mathrm{n}$ polynomial matrices in $\zeta$ and $\eta$ is denoted by $\mathbb{R}^{\mathrm{m} \times \mathrm{n}}[\zeta, \eta]$. A nonsingular matrix $R \in \mathbb{R}^{\mathrm{m} \times \mathrm{m}}[\xi]$ is Hurwitz if the roots of $\operatorname{det}(R)$ are all in the open left half-plane. The space of complex numbers whose real part is positive (negative) is denoted by $\mathbb{C}_{+}\left(\mathbb{C}_{-}\right)$. The space of complex vectors with $\mathrm{n}$ components is denoted by $\mathbb{C}^{\mathrm{n}}$. Given $A, B \in \mathbb{R}^{\bullet \times n}, \operatorname{col}(A, B)$ denotes the matrix obtained by stacking $A$ over $B$. For a function $f:[t-\epsilon, t) \rightarrow \mathbb{R}^{\bullet}$, with $\epsilon>0$, we define $f\left(t^{-}\right):=\lim _{\tau \nearrow t} f(\tau)$; and similarly for $f:(t, t+\epsilon] \rightarrow \mathbb{R}^{\bullet}$ we define $f\left(t^{+}\right):=\lim _{\tau \searrow t} f(\tau)$, provided that these limits exist.

\section{SWitched Linear DifFERENTIAL Systems (SLDS)}

In this note, we use standard concepts and notation of the behavioral setting, in particular those of linear differential behaviors, state maps, and quadratic differential forms. A simplified collection of the theory that is relevant for the presented results can be found in [10, p. 2046, App. Z]. We now recall the basic definitions of SLDS, see [10, p. 2039].

Definition 1: A switched linear differential system (SLDS) $\Sigma$ is a quadruple $\Sigma=\{\mathcal{P}, \mathcal{F}, \mathcal{S}, \mathcal{G}\}$ where $\mathcal{P}=\{1, \ldots, N\} \subset \mathbb{N}$, is the set of indices; $\mathcal{F}=\left\{\mathfrak{B}_{1}, \ldots, \mathfrak{B}_{N}\right\}$, with $\mathfrak{B}_{j} \in \mathfrak{C}^{\infty}\left(\mathbb{R}, \mathbb{R}^{w}\right)$ a linear differential behavior and $j \in \mathcal{P}$, is the bank of behaviors; $\mathcal{S} \subset\{s$ : $\mathbb{R} \rightarrow \mathcal{P}\}$, with $s$ piecewise constant and right-continuous, is the set of admissible switching signals; and $\mathcal{G}=\left\{\left(G_{k \rightarrow \ell}^{-}(\xi), G_{k \rightarrow \ell}^{+}(\xi)\right) \in\right.$ $\left.\mathbb{R}^{\bullet \times \mathrm{w}}[\xi] \times \mathbb{R}^{\bullet \times \mathrm{w}}[\xi] \mid 1 \leq k, \ell \leq N, k \neq \ell\right\}$, is the set of gluing conditions. The set of switching instants associated with $s \in \mathcal{S}$ is defined by $\mathbb{T}_{s}:=\left\{t \in \mathbb{R} \mid s\left(t^{-}\right) \neq s\left(t^{+}\right)\right\}=\left\{t_{1}, t_{2}, \ldots\right\}$, where $t_{i}<_{i+1}$.

The results in this note are valid for every admissible $s \in \mathcal{S}$ that is well-defined, i.e., for every finite time interval there exists only a finite number of switching instants.

Since $\mathfrak{B}_{j} \in \mathfrak{C}^{\infty}\left(\mathbb{R}, \mathbb{R}^{w}\right), j=1, \ldots, N$, it follows that the trajectories in $\mathfrak{B}^{\Sigma}$ are piecewise infinitely differentiable functions from $\mathbb{R}$ to $\mathbb{R}^{\mathrm{w}}$.

Definition 2: Let $\Sigma=\{\mathcal{P}, \mathcal{F}, \mathcal{S}, \mathcal{G}\}$ be a SLDS, and let $s \in \mathcal{S}$. The $s$ - switched linear differential behavior $\mathfrak{B}^{s}$ is the set of trajectories $w: \mathbb{R} \rightarrow \mathbb{R}^{\mathrm{w}}$ that satisfy the following two conditions:

1) for all $t_{i}, t_{i+1} \in \mathbb{T}_{s},\left.\left.w\right|_{\left[t_{i}, t_{i+1}\right)} \in \mathfrak{B}_{s\left(t_{i}\right)}\right|_{\left[t_{i}, t_{i+1}\right)}$;

2) $w$ satisfies the gluing conditions $\mathcal{G}$ at the switching instants for each $t_{i} \in \mathbb{T}_{s}$, i.e.,

$$
G_{s\left(t_{i}^{-}\right) \rightarrow s\left(t_{i}^{+}\right)}^{+}\left(\frac{d}{d t}\right) w\left(t_{i}^{+}\right)=G_{s\left(t_{i}^{-}\right) \rightarrow s\left(t_{i}^{+}\right)}^{-}\left(\frac{d}{d t}\right) w\left(t_{i}^{-}\right)
$$




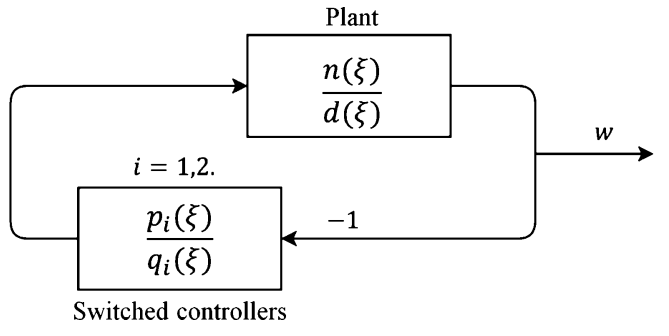

Fig. 1. Multi-controller system.

The switched linear differential behavior $(S L D B) \mathfrak{B}^{\Sigma}$ of $\Sigma$ is defined by $\mathfrak{B}^{\Sigma}:=\bigcup_{s \in \mathcal{S}} \mathfrak{B}^{s}$.

The gluing conditions describe constraints on the trajectories of the external variable at switching instants, e.g., charge/flux conservation principles, kinematic constraints, reset maps, etc. When dealing with autonomous modes, equivalent gluing conditions can be written in terms of the state (see [10, Sec. II-B]). In the following, we focus on the study of SLDS with a standard type of gluing conditions that are written in terms of states constructed from the linear differential equations describing the modes. In order to do so, we use the notion of state maps that act on the external variable (see [10, p. 2047, App. A-C]). We also use the concept of $R$-canonical representative for a polynomial matrix $G \in \mathbb{R}^{\bullet \times \mathrm{w}}[\xi]$ denoted by $G$ $\bmod R$ (see [10, p. 2047, App. A-B]). We recall the following instrumental result.

Lemma 1: Let $\mathfrak{B}_{i}=\operatorname{ker} R_{i}(d / d t), i=1,2$. Assume that $R_{1}, R_{2} \in$ $\mathbb{R}^{\mathrm{w} \times \mathrm{w}}[\xi]$ are nonsingular, and that $R_{2} R_{1}^{-1}$ is strictly proper. Let $n_{i}:=$ $\operatorname{deg}\left(\operatorname{det}\left(R_{i}\right)\right), \quad i=1,2$. There exist $X_{1}^{\prime} \in \mathbb{R}^{\left(n_{1}-n_{2}\right) \times \mathrm{w}}[\xi], X_{2} \in$ $\mathbb{R}^{n_{2} \times \mathrm{w}}[\xi]$ such that $X_{2}(d / d t)$ is a minimal state map for $\mathfrak{B}_{2}$, and $X_{1}(d / d t):=\left[\begin{array}{ll}X_{2}(d / d t) & X_{1}^{\prime}(d / d t)\end{array}\right]^{\top}$, is a minimal state map for $\mathfrak{B}_{1}$. Moreover, there exists $\Pi \in \mathbb{R}^{\left(n_{1}-n_{2}\right) \times n_{2}}$ such that $X_{1}^{\prime}(\xi)$ $\bmod R_{2}=\Pi X_{2}(\xi)$.

Proof: See [10], Lemma 1.

Definition 3: Let $\Sigma$ be a SLDS with mode behaviors $\mathfrak{B}_{i}:=$ ker $R_{j}(d / d t), j=1,2$, where $R_{j} \in \mathbb{R}^{\mathrm{w} \times \mathrm{w}}[\xi], j=1,2$, is Hurwitz and nonsingular. Assume that $R_{2} R_{1}^{-1}$ is strictly proper. Let $n_{i}:=\operatorname{deg}\left(\operatorname{det}\left(R_{i}\right)\right), i=1,2$, and let $X_{1}^{\prime} \in \mathbb{R}^{\left(n_{1}-n_{2}\right) \times \mathrm{w}}[\xi], X_{2} \in$ $\mathbb{R}^{n_{2} \times \mathrm{w}}[\xi]$ and $\Pi \in \mathbb{R}^{\left(n_{1}-n_{2}\right) \times n_{2}}$ be as in Lemma $1 . \Sigma$ is a standard $S L D S$ if the gluing conditions are

$$
\begin{aligned}
& \left(G_{2 \rightarrow 1}^{-}(\xi), G_{2 \rightarrow 1}^{+}(\xi)\right):=\left(\operatorname{col}\left(X_{2}(\xi), \Pi X_{2}(\xi)\right), \operatorname{col}\left(X_{2}(\xi), X_{1}^{\prime}(\xi)\right)\right) \\
& \left(G_{1 \rightarrow 2}^{-}(\xi), G_{1 \rightarrow 2}^{+}(\xi)\right):=\left(X_{2}(\xi), X_{2}(\xi)\right) .
\end{aligned}
$$

Example 1: Consider the basic multi-controller system in Fig. 1, where the plant described by the transfer function $n(\xi) / d(\xi):=$ $((\xi+1)(\xi+4)) /((\xi-2)(\xi+3))$ is interconnected to stabilising switched controllers described by $p_{1}(\xi) / q_{1}(\xi):=\left(K_{D} \xi^{2}+K_{P} \xi+\right.$ $\left.K_{I}\right) / \xi$ and $p_{2}(\xi) / q_{2}(\xi):=\left(K_{P}^{\prime} \xi+K_{I}^{\prime}\right) / \xi$, where $K_{D}=1, K_{P}=$ $25, K_{I}=150, K_{P}^{\prime}=1$, and $K_{I}^{\prime}=33$.

By selecting the output variable $w$ as the variable of interest, we can model the mode behaviors as $\mathfrak{B}_{i}:=\operatorname{ker} r_{i}(d / d t), i=$ 1,2 , with $r_{1}(\xi):=600+844 \xi+280 \xi^{2}+31 \xi^{3}+\xi^{4}$ and $r_{2}(\xi):=$ $132+163 \xi+39 \xi^{2}+2 \xi^{3}$. Furthermore, in many cases we are interested in determining a re-initialization for the controllers at switching instants that guarantees the continuity of the external variable, i.e., $w\left(t_{j}^{+}\right)=w\left(t_{j}^{-}\right)$for all $t_{j} \in \mathbb{T}_{s}$ (see e.g., the bumpless transfer problem in [11]). We model such requirements via gluing conditions, i.e., when switching from $\mathfrak{B}_{1}$ to $\mathfrak{B}_{2}$ at $t_{j}$, we consider

$$
\left[\begin{array}{c}
w\left(t_{j}^{+}\right) \\
\frac{d}{d t} w\left(t_{j}^{+}\right) \\
\frac{d^{2}}{d t^{2}} w\left(t_{j}^{+}\right)
\end{array}\right]=\left[\begin{array}{c}
w\left(t_{j}^{-}\right) \\
\frac{d}{d t} w\left(t_{j}^{-}\right) \\
\frac{d^{2}}{d t^{2}} w\left(t_{j}^{-}\right)
\end{array}\right] .
$$

On the other hand, when we switch from from $\mathfrak{B}_{2}$ to $\mathfrak{B}_{1}$, we consider

$$
\left[\begin{array}{c}
w\left(t_{j}^{+}\right) \\
\frac{d}{d t} w\left(t_{j}^{+}\right) \\
\frac{d^{2}}{d t^{2}} w\left(t_{j}^{+}\right) \\
\frac{d^{3}}{d t^{3}} w\left(t_{j}^{+}\right)
\end{array}\right]=\left[\begin{array}{c}
w\left(t_{j}^{-}\right) \\
\frac{d}{d t} w\left(t_{j}^{-}\right) \\
\frac{d^{2}}{d t^{2}} w\left(t_{j}^{-}\right) \\
-66 w\left(t_{j}^{-}\right)-\frac{163}{2} \frac{d}{d t} w\left(t_{j}^{-}\right)-\frac{39}{2} \frac{d^{2}}{d t^{2}} w\left(t_{j}^{-}\right)
\end{array}\right]
$$

The rationale underlying this choice of gluing conditions is that at switching instants any trajectory of $\mathfrak{B}_{1}$ and $\mathfrak{B}_{2}$ is uniquely specified by the instantaneous values of $w$ and its derivatives, respecting the laws imposed by the mode behaviors and requiring that the values of $w\left(t_{j}^{+}\right)$and $w\left(t_{j}^{-}\right)$coincide. Since $X_{1}(\xi):=\left[\begin{array}{llll}1 & \xi & \xi^{2} & \xi^{3}\end{array}\right]^{\top}$ and $X_{2}(\xi):=\left[\begin{array}{lll}1 & \xi & \xi^{2}\end{array}\right]^{\top}$ induce state maps for $\mathfrak{B}_{1}$ and $\mathfrak{B}_{2}$ respectively, note that the proposed gluing conditions are standard in the sense of Definition 3, where $\Pi=[-66-(163 / 2)-(39 / 2)]$.

Remark 1: Standard gluing conditions describe concatenability specifications when switching between mode behaviors with different state space dimensions as in Ex. 1. In more complex cases, e.g., a multivariable version of Ex. 1, standard gluing conditions can be computed using Lemma 1. Standard gluing conditions also appear in switched electrical systems, see e.g., the example of the energy distribution network presented in $[9, \mathrm{Sec} . \mathrm{V}]$.

\section{Stability And Positive-Realness}

A SLDS $\Sigma$ is asymptotically stable if $\lim _{t \rightarrow \infty} w(t)=0$ for all $w \in$ $\mathfrak{B}^{\Sigma}$. We prove asymptotic stability of a SLDS showing the existence of a Lyapunov function $Q_{\Psi}$, i.e., a QDF such that is a Lyapunov function for the individual modes, i.e., $Q_{\Psi} \stackrel{\mathfrak{B}_{k}}{\geq} 0$ and $(d / d t) Q_{\Psi} \stackrel{\mathfrak{B}_{k}}{<} 0$, $k=1, \ldots, N$, (as in [19, Th. 4.3]; moreover, see [19, Proposition 4.7] to verify that, if exists, $Q_{\Psi}$ is such that $Q_{\Psi} \stackrel{\mathfrak{B}_{k}}{>} 0$ ). Moreover the value of $Q_{\Psi}$ does not increase at the switching instants, i.e., $Q_{\Psi}(w)\left(t_{i}^{-}\right) \geq$ $Q_{\Psi}(w)\left(t_{i}^{+}\right)$for all $t_{i} \in \mathbb{T}_{s}$, see [10, Th. 1]. We also use the concept of $R$-canonical representative for QDFs, see [10, p. 2047, App. A-D]. We now recall an important structural property of a Lyapunov function for a standard SLDS.

Lemma 2: Let $\Sigma$ be a standard SLDS as in Definition 3. Consider a two-variable polynomial matrix

$$
\Psi(\zeta, \eta)=\left[\begin{array}{ll}
X_{2}(\zeta)^{\top} & X_{1}^{\prime}(\zeta)^{\top}
\end{array}\right] \underbrace{\left[\begin{array}{ll}
\Psi_{11} & \Psi_{12} \\
\Psi_{12}^{\top} & \Psi_{22}
\end{array}\right]}_{=: \widetilde{\Psi}}\left[\begin{array}{l}
X_{2}(\eta) \\
X_{1}^{\prime}(\eta)
\end{array}\right]
$$

where $\quad \Psi_{11} \in \mathbb{R}^{n_{2} \times n_{2}}, \quad \Psi_{12} \in \mathbb{R}^{n_{2} \times\left(n_{1}-n_{2}\right)} \quad$ and $\quad \Psi_{22} \in$ $\mathbb{R}^{\left(n_{1}-n_{2}\right) \times\left(n_{1}-n_{2}\right)}$. Assume that $\widetilde{\Psi}>0$. The condition $Q_{\Psi}(w)\left(t_{i}^{-}\right) \geq$ $Q_{\Psi}(w)\left(t_{i}^{+}\right)$is satisfied for all $t_{i} \in \mathbb{T}_{s}$ and for all $w \in \mathfrak{B}^{\Sigma}$, if and only if $\Psi_{12}=-\Pi^{\top} \Psi_{22}$.

Proof: See [10, p. 2048, Lemma 3].

We call a matrix of rational functions $G(\xi)$ strictly positive-real if it is analytic in $\mathbb{C}_{+}$and $G(-j \omega)^{\top}+G(j \omega)>0 \forall \omega \in \mathbb{R}$. In the following theorem we show that strict positive-realness is a sufficient condition for the asymptotic stability of standard SLDS.

Theorem 1: Let $\Sigma$ be a standard SLDS as in Definition 3. Assume that $R_{2} R_{1}^{-1}$ is strictly positive-real. There exists $Q \in$ $\mathbb{R}^{\bullet \times w}[\xi]$ such that $R_{1}(-\xi)^{\top} R_{2}(\xi)+R_{2}(-\xi)^{\top} R_{1}(\xi)=Q(-\xi)^{\top}$ $Q(\xi)$, rank $\operatorname{col}\left(R_{1}(\lambda), Q(\lambda)\right)=\mathrm{w}$ for all $\lambda \in \mathbb{C}$ and $Q R_{1}^{-1}$ is strictly proper. Define

$$
\Psi(\zeta, \eta):=\frac{R_{1}(\zeta)^{\top} R_{2}(\eta)+R_{2}(\zeta)^{\top} R_{1}(\eta)-Q(\zeta)^{\top} Q(\eta)}{\zeta+\eta} .
$$


Then $\Psi$ is $R_{1}$-canonical; and induces a QDF $Q_{\Psi}$ which is a Lyapunov function for $\Sigma$.

Proof: See [10, Th. 4].

Theorem 1 reduces the computation of a Lyapunov function for standard SLDS to the computation of polynomial spectral factors $Q \in \mathbb{R}^{\bullet \times w}[\xi]$, whose existence follow from positive-realness [1].

Remark 2: In Theorem 1, we show a different perspective when compared to existing results (e.g., [6], [16], [17]) on the relation between positive-realness and stability: the dynamical regimes do not arise from closing the loop around some fixed plant. Positive-realness arises from the interplay of the mode dynamicals, i.e., the construction of a rational matrix involving the two modes.

Example 2 (Continued From Example 1): Note that since $r_{1}(-j \omega)$ $r_{2}(j \omega)+r_{2}(-j \omega) r_{1}(j \omega)>0$ for all $\omega \in \mathbb{R}$, we conclude that $r_{2}(\xi) / r_{1}(\xi)$ is strictly positive real. Consequently, using Theorem 1 , we also conclude that the standard SLDS is asymptotically stable under arbitrary swiching signals.

The following results enable an alternative computation of $\Psi$ and $Q$ as in Theorem 1 using an easy-to-contruct matrix equation.

Proposition 1: Consider the assumptions in Theorem 1. Define $n_{1}:=\operatorname{deg}\left(\operatorname{det}\left(R_{1}\right)\right)$ and let $X_{1} \in \mathbb{R}^{n_{1} \times \mathrm{w}}[\xi]$ be a minimal state map for $\mathfrak{B}_{1}$. Write $R_{1}(\xi)=\sum_{j=0}^{L} R_{1, j} \xi^{j}$, with $R_{1, j} \in \mathbb{R}^{\mathrm{w} \times \mathrm{w}}, j=0$, $1, \ldots, L$. There exists $\widetilde{R}_{2} \in \mathbb{R}^{\mathrm{w} \times n_{1}}, \widetilde{Q} \in \mathbb{R}^{\bullet \times n_{1}}$ and $\widetilde{\Psi} \in \mathbb{R}^{n_{1} \times n_{1}}$ such that $R_{2}(\xi)=\widetilde{R}_{2} X_{1}(\xi), \quad Q(\xi)=\widetilde{Q} X_{1}(\xi)$ and $\Psi(\zeta, \eta)=$ $X_{1}(\zeta)^{\top} \widetilde{\Psi} X_{1}(\eta)$. Moreover, there exist $X_{1, j} \in \mathbb{R}^{n_{1} \times \mathrm{w}}$, with $j=0,1$, $\ldots, L-1$, such that $X_{1}(\xi)=\sum_{j=0}^{L-1} X_{1, j} \xi^{j}$.

Proof: See Appendix.

Proposition 2: Consider the assumptions of Theorem 1 and the results obtained in Propostion 1. Denote the coefficient matrices of $R_{1}(\xi)$ and $X_{1}(\xi)$ by $\widetilde{R}_{1}:=\left[\begin{array}{lll}R_{1,0} & \ldots & R_{1, L}\end{array}\right]$, and $\widetilde{X}_{1}:=$ $\left[\begin{array}{lll}X_{1,0} & \ldots & X_{1, L-1}\end{array}\right]$. Let $\widetilde{\Psi}=\widetilde{\Psi}^{\top} \in \mathbb{R}^{n_{1} \times n_{1}}$. The following statements are equivalent:

1) $\Psi(\zeta, \eta):=X_{1}(\zeta)^{\top} \widetilde{\Psi} X_{1}(\eta), \quad R_{i}(\xi), \quad i=1, \quad 2, \quad$ and $\quad Q(\xi)$ satisfy (3);

2) There exists $\widetilde{\Psi}>0$, such that

$$
\begin{aligned}
& {\left[\begin{array}{c}
0_{\mathrm{w} \times n_{1}} \\
\widetilde{X}_{1}^{\top}
\end{array}\right] \widetilde{\Psi}\left[\begin{array}{cc}
\widetilde{X}_{1} & 0_{n_{1} \times \mathrm{w}}
\end{array}\right]+\left[\begin{array}{c}
\widetilde{X}_{1}^{\top} \\
0_{\mathrm{w} \times n_{1}}
\end{array}\right] \widetilde{\Psi}\left[\begin{array}{ll}
0_{n_{1} \times \mathrm{w}} & \widetilde{X}_{1}
\end{array}\right]-\left[\begin{array}{c}
\widetilde{X}_{1}^{\top} \\
0_{\mathrm{w} \times n_{1}}
\end{array}\right]} \\
& \times \widetilde{R}_{2}^{\top} \widetilde{R}_{1}-\widetilde{R}_{1}^{\top} \widetilde{R}_{2}\left[\begin{array}{ll}
\widetilde{X}_{1} & 0_{n_{1} \times \mathrm{w}}
\end{array}\right]+\left[\begin{array}{c}
\widetilde{X}_{1}^{\top} \\
0_{\mathrm{w} \times n_{1}}
\end{array}\right] \widetilde{Q}^{\top} \widetilde{Q}\left[\begin{array}{ll}
\widetilde{X}_{1} & 0_{n_{1} \times \mathrm{w}}
\end{array}\right]=0 .
\end{aligned}
$$

Proof: See Appendix.

The result provided in Proposition 2 permits and easy test of asymptotic stability of standard SLDS, which is an straightforward matter for standard LMI solvers.

Remark 3: Strict positive-realness of $R_{2} R_{1}^{-1}$ in Theorem 1 implies the existence of a Lyapunov function for standard SLDS. However, it can be easily proved that the converse implication is not true in general.

\section{Positive-Real Completions}

We now study the role of positive-real completions in stability of standard SLDS.

Definition 4: Let $R_{i} \in \mathbb{R}^{\mathrm{w} \times \mathrm{w}}[\xi], i=1,2$ be nonsingular and $R_{2} R_{1}^{-1}$ strictly proper. $M \in \mathbb{R}^{\mathrm{w} \times \mathrm{w}}[\xi]$ is a strictly positive-real completion of $R_{2} R_{1}^{-1}$ if $M R_{2} R_{1}^{-1}$ is strictly positive-real.

Remark 4: A positive-real completion can be regarded as the multivariable version of the "passivation" technique used for open SISO systems in $[5, \mathrm{Sec}$. III]. We will show that in the context of SLDS, positive-real completions provide a less conservative stability condition than that of Theorem 1, i.e., for the case when $R_{2} R_{1}^{-1}$ is not positive-real.

Remark 5: Strictly- positive-real completions are not unique, e.g., the rational function $m r_{2} / r_{1}$ with $r_{1}(\xi):=(\xi+1)(\xi+3)(\xi+6)$ and $r_{2}(\xi):=\xi+2$ is positive-real with $m$ equal to $\xi+4, \xi+5$ or many other. Note also that not every pair of Hurwitz matrices has a strictly- positive-real completion, for example the polynomials $r_{1}(\xi):=2523677+435616 \xi+81559 \xi^{2}+7000 \xi^{3}+603 \xi^{4}+$ $24 \xi^{5}+\xi^{6}$ and $r_{2}(\xi):=65+46 \xi+26 \xi^{2}+6 \xi^{3}+\xi^{4}$ have not.

In the following result, we establish general conditions for the existence of a Lyapunov function for a standard SLDS using positivereal completions.

Theorem 2: Let $\Sigma$ be a standard SLDS as in Definition 3. Let $\widetilde{R}_{1}$ and $\widetilde{X}_{1}$ be as in Proposition 2. Define $Y:=M R_{2}$ with $M \in \mathbb{R}^{\mathrm{w} \times \mathrm{w}}[\xi]$ such that $Y R_{1}^{-1}$ is strictly proper. There exist $Y_{j} \in \mathbb{R}^{\mathrm{w} \times \mathrm{w}}$, with $j=0,1, \ldots, L-1$, such that $Y(\xi)=\sum_{j=0}^{L-1} Y_{j} \xi^{j}$. Denote $\tilde{Y}:=$ $\left[Y_{0} \cdots Y_{L-1}\right]$. If there exists $\widetilde{\Psi}>0$ with $\widetilde{\Psi} \in \mathbb{R}^{n_{1} \times n_{1}}$ such that

$$
\begin{aligned}
{\left[\begin{array}{c}
0_{\mathrm{w} \times n} \\
\widetilde{X}_{1}^{\top}
\end{array}\right] \widetilde{\Psi}\left[\widetilde{X}_{1} \quad 0_{n \times \mathrm{w}}\right]+\left[\begin{array}{c}
\widetilde{X}_{1}^{\top} \\
0_{\mathrm{w} \times n}
\end{array}\right] \widetilde{\Psi}\left[0_{n \times \mathrm{w}} \quad \widetilde{X}_{1}\right] } \\
\\
\quad-\left[\begin{array}{c}
\widetilde{Y}^{\top} \\
0_{\mathrm{w} \times \mathrm{w}}
\end{array}\right] \widetilde{R}_{1}-\widetilde{R}_{1}^{\top}\left[\begin{array}{ll}
\widetilde{Y} & 0_{\mathrm{w} \times \mathrm{w}}
\end{array}\right] \leq 0
\end{aligned}
$$

then $M$ is a strictly positive-real completion of $R_{2} R_{1}^{-1}$. Moreover, if $\widetilde{\Psi}$ partitioned as in (2) is such that $\Psi_{12}=-\Pi^{\top} \Psi_{22}$, then $X_{1}(\zeta)^{\top} \widetilde{\Psi} X_{1}(\eta)$ induces a Lyapunov function for $\Sigma$.

Proof: See Appendix.

Remark 6: Theorem establishes general conditions for stability of standard SLDS in terms of LMIs. Although positive-real completions are instrumental for the computations, they are unknown in general; however, they can be computed using the LMI (4). In order to do so, let $M(\xi)=\sum_{j=0}^{N} M_{j \xi^{j}}$, i.e., $M(\xi)$ is written in terms of unspecified parameters, with $N \leq L-1$. Write

$$
\tilde{Y}^{\top}:=\underbrace{\left[\begin{array}{ccccc}
R_{2,0} & 0 & 0 & \cdots & 0 \\
R_{2,1} & R_{2,0} & 0 & \cdots & 0 \\
\vdots & \vdots & \ddots & \cdots & \vdots
\end{array}\right]}_{=: \widetilde{T}} \underbrace{\left[\begin{array}{c}
M_{0} \\
M_{1} \\
\vdots
\end{array}\right]}_{=: \widetilde{M}}
$$

where $\widetilde{T} \in \mathbb{R}^{L \times \bullet}$ is a block Töplitz matrix (see [4, Sec. 8.3.1]) containing the coefficients $R_{2, j}$ of $R_{2}(\xi)$; and $\widetilde{M} \in \mathbb{R}^{\bullet \times w}$ contains the unknown coefficients of $M(\xi)$. The LMI (4) with $\widetilde{Y}$ as in (5) can be solved using standard LMI solvers. On the other hand, if (4) has no solution, we conclude that the pair $R_{1}, R_{2}$ does not have a positive-real completion, see remark 5.

Remark 7: We have focused on the study of standard SLDS whose dynamic modes are associated to $R_{2} R_{1}^{-1}$ being strictly proper. It is also possible to extend these results to the case when $R_{2} R_{1}^{-1}$ is biproper and show that positive-realness is also a sufficient condition for this case. This has been already shown for the case $\mathrm{w}=1$ in [12]. A positive-real completion can be also computed in the proper case, e.g., using the positive-real lemma for the state space realisation of $M R_{2} R_{1}^{-1}$.

\section{Stability of Standard Slds With Augmented Banks}

In this section, we analyze important consequences of the existence of positive-real completions. The following lemma will be instrumental for this aim.

Lemma 3: Let $\mathfrak{B}_{i}:=\operatorname{ker} R_{i}(d / d t), i=1,2$, be as in Definition 3. Let $M \in \mathbb{R}^{\mathrm{w} \times \mathrm{w}}[\xi]$ be such that $M R_{2} R_{1}^{-1}$ is strictly proper. 
Define $\mathfrak{B}_{3}:=\operatorname{ker} R_{3}(d / d t)$ where $R_{3}(\xi):=M(\xi) R_{2}(\xi)$ and $n_{j}:=$ $\operatorname{deg}\left(\operatorname{det}\left(R_{j}\right)\right), j=1,2$, 3. There exist $X_{2} \in \mathbb{R}^{n_{2} \times \mathrm{w}}[\xi], \quad X_{3}^{\prime} \in$ $\mathbb{R}^{\left(n_{3}-n_{2}\right) \times \mathrm{w}}[\xi]$ and $X_{1}^{\prime} \in \mathbb{R}^{\left(n_{1}-n_{3}\right) \times \mathrm{w}}[\xi]$ such that:

1) $X_{1}:=\left[\begin{array}{lll}X_{2} & X_{3}^{\prime} & X_{1}^{\prime}\end{array}\right]^{\top}$ is a minimal state map for $\mathfrak{B}_{1}$.

2) $X_{2}$ is a minimal state map for $\mathfrak{B}_{2}$.

3) $X_{3}:=\left[\begin{array}{ll}X_{2} & X_{3}^{\prime}\end{array}\right]^{\top}$ is a minimal state map for $\mathfrak{B}_{3}$.

Moreover, there exist $\Pi_{j}, j=1,2$, 3, of adequate sizes, such that $\operatorname{col}\left(X_{3}^{\prime}(\xi), X_{1}^{\prime}(\xi)\right) \bmod R_{2}=\Pi_{1} X_{2}(\xi) ; X_{3}^{\prime}(\xi) \bmod R_{2}=$ $\Pi_{2} X_{2}(\xi)$; and $X_{1}^{\prime}(\xi) \bmod R_{3}=\Pi_{3} X_{3}(\xi)$.

Proof: See Appendix.

In the following, we show a sufficient condition for the asymptotic stability of a SLDS with three behaviors.

Theorem 3: Let $\Sigma$ be a standard SLDS as in Definition 3. Assume that there exists $M$ and $\widetilde{\Psi}$ satisfying the conditions of Theorem. Define $R_{3}:=M R_{2}, \mathfrak{B}_{i}:=\operatorname{ker} R_{i}(d / d t), i=1,2,3$; and let $X_{i}, i=1,2,3$ be as in Lemma 3. Consider a SLDS $\Sigma^{\prime}$ with $\mathcal{F}^{\prime}=\left\{\mathfrak{B}_{1}, \mathfrak{B}_{2}, \mathfrak{B}_{3}\right\}$ and gluing conditions

$$
\begin{aligned}
& \left(G_{2 \rightarrow 1}^{-}(\xi), G_{2 \rightarrow 1}^{+}(\xi)\right):=\left(\operatorname{col}\left(X_{2}(\xi), \Pi_{1} X_{2}(\xi)\right), \operatorname{col}\left(X_{3}(\xi), X_{1}^{\prime}(\xi)\right)\right) \\
& \left(G_{1 \rightarrow 2}^{-}(\xi), G_{1 \rightarrow 2}^{+}(\xi)\right):=\left(X_{2}(\xi), X_{2}(\xi)\right) \\
& \left(G_{3 \rightarrow 1}^{-}(\xi), G_{3 \rightarrow 1}^{+}(\xi)\right):=\left(\operatorname{col}\left(X_{3}(\xi), \Pi_{3} X_{3}(\xi)\right), \operatorname{col}\left(X_{3}(\xi), X_{1}^{\prime}(\xi)\right)\right) \\
& \left(G_{1 \rightarrow 3}^{-}(\xi), G_{1 \rightarrow 3}^{+}(\xi)\right):=\left(X_{3}(\xi), X_{3}(\xi)\right) \\
& \left(G_{2 \rightarrow 3}^{-}(\xi), G_{2 \rightarrow 3}^{+}(\xi)\right):=\left(\operatorname{col}\left(X_{2}(\xi), \Pi_{2} X_{2}(\xi)\right), \operatorname{col}\left(X_{2}(\xi), X_{3}^{\prime}(\xi)\right)\right) \\
& \left(G_{3 \rightarrow 2}^{-}(\xi), G_{3 \rightarrow 2}^{+}(\xi)\right):=\left(X_{2}(\xi), X_{2}(\xi)\right)
\end{aligned}
$$

with $\Pi_{i}, i=1,2,3$ as in Lemma 3 . Then $X_{1}(\zeta)^{\top} \widetilde{\Psi} X_{1}(\eta)$ induces a Lyapunov function for $\mathcal{F}^{\prime}$.

Proof: See Appendix.

Another consequence of the notion of positive-real completion is given in the following theorem, where we prove stability of parameter dependent families of SLDS with three behaviors.

Theorem 4: Let $\Sigma$ be a standard SLDS as in Definition 3. Theorem 3. Assume that there exist strictly positive-real completions $M_{1}$ and $M_{2}$ of $R_{2} R_{1}^{-1}$, each one associated to a Lyapunov function for $\Sigma$ as in Theorem. Then, the polynomial matrix $M_{\alpha}:=\alpha M_{1}+(1-\alpha) M_{2}$ is also a strictly positive-real completion for every $\alpha$ such that $0 \leq \alpha \leq$ 1. Moreover, define a SLDS $\Sigma^{\prime}$ with

$$
\mathcal{F}_{\alpha}^{\prime}:=\left\{\operatorname{ker} R_{1}\left(\frac{d}{d t}\right), \operatorname{ker} R_{2}\left(\frac{d}{d t}\right), \operatorname{ker} R_{3, \alpha}\left(\frac{d}{d t}\right)\right\}
$$

where $R_{3, \alpha}:=M_{\alpha} R_{2}$, and with gluing conditions as in Theorem 3 . Then $\Sigma^{\prime}$ is asymptotically stable.

Proof: See the Appendix.

\section{CONCLUSION}

We provided general stability conditions for standard SLDS using the notion of positive realness in Theorems 1 and 2. In Theorem 3, we showed that the existence of a strictly positive-real completion $M$ associated to a standard SLDS $\Sigma$, implies the existence of a third behavior $\mathfrak{B}_{3}:=\operatorname{ker} M R_{2}(d / d t)$, in an augmented bank $\mathcal{F}^{\prime}$ of a SLDS $\Sigma^{\prime}$. We defined standard gluing conditions for $\Sigma^{\prime}$, associated to the switching among the behaviors $\mathfrak{B}_{i}, i=1,2,3$. Consequently, the stability conditions derived from the analysis of the switching between the behaviors in $\mathcal{F}$ are analogous to those for $\mathcal{F}^{\prime}$ concluding that if $\Sigma$ is asymptotically stable so is $\Sigma^{\prime}$. In Theorem 4 we showed that the existence of two separate completions allows to establish the stability of a whole family of parameter-dependent SLDS with three behaviors $\mathcal{F}_{\alpha}^{\prime}$. We also showed that the asymptotic stability of a completion established in Theorem 3 is in a sense "robust": perturbations of a given completion, parametrized by $\alpha$ as in Theorem 4 , also result in a stable SLDS. For further results regarding LMI-based stability conditions of SLDS with multiple modes using multiple higher-order Lyapunov functions, the reader can be referred to [10].

\section{APPENDIX}

Proof of Proposition 1: The existence of $\widetilde{R}_{2}$ and $\widetilde{Q}$ follows from the fact that $R_{2} R_{1}^{-1}$ and $Q R_{1}^{-1}$ are strictly proper and that the rows of $X_{1}(\xi)$ are a basis of the vector space over $\mathbb{R}$ defined by $\left\{f \in \mathbb{R}^{1 \times w}[\xi] \mid f R^{-1}\right.$ is strictly proper $\}$. Using this argument and the fact that $\Psi$ is $R_{1}$-canonical, we also conclude that $\widetilde{\Psi}$ exists. The claim that the highest degree present in $X_{1}$ is less than that in $R_{1}$, follows from the strict properness oft $X R_{1}^{-1}$ and [3, Lemma 6.3-10].

Proof of Proposition 2: Considering Proposition 1, the proof is analogous to that in [10, Prop. 1, p. 2024].

Proof of Theorem: Following the same arguments used in the proofs of Propositions 1 and 2, it can be verified that the LMI (4) is equivalent to

$$
\Psi(\zeta, \eta)=R_{1}(\zeta)^{\top} Y(\eta)+Y(\zeta)^{\top} R_{1}(\eta)-D(\zeta)^{\top} D(\eta)
$$

for some $D \in \mathbb{R}^{\bullet \times w}[\xi]$ which implies that $Y R_{1}^{-1}=M R_{2} R_{1}^{-1}$ is strictly positive-real. To prove the final claim note that according to Theorem $1, Q_{\Psi}=X_{1}(d / d t)^{\top} \widetilde{\Psi} X_{1}(d / d t)$ is a Lyapunov function for $\mathfrak{B}_{1}$. Thus, to prove that $Q_{\Psi}$ is also a Lyapunov function for $\mathfrak{B}_{2}$ define $\Psi_{2}(\zeta, \eta):=\Psi(\zeta, \eta) \bmod R_{2}$. Note that since $Q_{\Psi} \geq 0$ and $Q_{\Psi} \stackrel{\mathfrak{B}_{2}}{=} Q_{\Psi_{2}}$, it follows that $Q_{\Psi_{2}} \geq 0$. In order to prove that $(d / d t) Q_{\Psi} \stackrel{\mathfrak{B}_{2}}{<} 0$ it is enough to show that $\operatorname{col}\left(D(\lambda), R_{2}(\lambda)\right)$ is full column rank for all $\lambda \in \mathbb{C}$. By contradiction, assume that there exists $\lambda \in \mathbb{C}$ and $v \in \mathbb{C}^{\mathrm{w}}$ such that $R_{2}(\lambda) v=0$ and $Q(\lambda) v=0$. Note that since $R_{2}$ is Hurwitz it follows that $\lambda \in \mathbb{C}_{-}$, the open left half-plane. Substitute $\zeta=\bar{\lambda}$ and $\eta=\lambda$ in the expression in (6), obtaining $(\bar{\lambda}+$ $\lambda) \Psi(\bar{\lambda}, \lambda)=0$. Since $\lambda \in \mathbb{C}_{-}$, this is equivalent to $\Psi(\bar{\lambda}, \lambda)=0$, which implies that $Q_{\Psi}$ is not positive-definite, a contradiction. Finally, the condition $Q_{\Psi}(w)\left(t_{i}^{-}\right) \geq Q_{\Psi}(w)\left(t_{i}^{+}\right)$follows from the fact that $\Psi_{12}=-\Pi^{\top} \Psi_{22}$ and Lemma 2 .

Proof of Lemma 3: We know from Lemma 1 that the polynomial row vectors that form a basis for the state space of $\mathfrak{B}_{2}$ are contained in that of $\mathfrak{B}_{1}$. We can arrange such vectors that form a minimal state map $X_{2}$ in the first $n_{2}$-rows of $X_{1}$. Moreover, since $R_{3} R_{1}^{-1}$ is strictly proper, we apply the same argument to arrange in the first $n_{3}$-rows, the vectors that form a basis for the state space of $\mathfrak{B}_{3}$ including those in $X_{2}$ and the additional $\left(n_{3}-n_{2}\right)$-vectors, denoted by $X_{3}^{\prime}$. The existence of $\Pi_{i}, i=1,2,3$ follows from the same argument used in Lemma 1.

Proof of Theorem 3: In order to show that $Q_{\Psi}$ is a Lyapunov function for $\mathcal{F}^{\prime}$, we prove the following statements. S1: $Q_{\Psi} \stackrel{\mathfrak{B}_{1}}{\geq} 0$ and $(d / d t) Q_{\Psi} \stackrel{\mathfrak{B}_{1}}{<} 0 ; \mathbf{S 2}: Q_{\Psi} \stackrel{\mathfrak{B}_{2}}{\geq} 0$ and $(d / d t) Q_{\Psi} \stackrel{\mathfrak{B}_{2}}{<} 0 ; \mathbf{S 3}: Q_{\Psi} \stackrel{\mathfrak{B}_{3}}{\geq} 0$ and $(d / d t) Q_{\Psi} \stackrel{\mathfrak{B}_{3}}{<} 0$. Moreover, we prove that the value of $Q_{\Psi}$ does not increase when switching between: $\mathbf{S 4}: \mathfrak{B}_{1}$ and $\mathfrak{B}_{2} ; \mathbf{S 5}: \mathfrak{B}_{1}$ and $\mathfrak{B}_{3}$; and S6: $\mathfrak{B}_{3}$ and $\mathfrak{B}_{2}$.

Note that statements $\mathbf{S 1}$ and $\mathbf{S} 2$ and $\mathbf{S} \mathbf{4}$ hold, since $Q_{\Psi}$ is a Lyapunov function for a standard SLDS with mode behaviors $\left\{\mathfrak{B}_{1}, \mathfrak{B}_{2}\right\}$. The validity of statement $\mathbf{S 5}$ follows from Theorem 1 , since $R_{3} R_{1}^{-1}$ is strictly positive-real. The proof of $\mathbf{S 3}$, follows from defining $\Psi_{3}(\zeta, \eta):=$ $\Psi(\zeta, \eta) \bmod R_{3}$ and applying the same arguments used in the proof of Theorem for $\Psi(\zeta, \eta) \bmod R_{2}$. It now remains to prove S6. Consider the following lemma. 
Lemma 4: Let $X_{1}, R_{2}, R_{3}$ and $\Pi_{i}, i=1,2,3$, be as in the theorem, then $\left(X_{1} \bmod R_{3}\right) \bmod R_{2}=X_{1} \bmod R_{2}$. Moreover, considering the partition $\Pi_{3}:=\left[\Pi_{3}^{\prime} \Pi_{3}^{\prime \prime}\right]$ with $\Pi_{3}^{\prime} \in \mathbb{R}^{\left(n_{1}-n_{3}\right) \times n_{2}}$ and $\Pi_{3}^{\prime \prime} \in \mathbb{R}^{\left(n_{1}-n_{3}\right) \times\left(n_{3}-n_{2}\right)}$, it follows that $\Pi_{1}=\left[\begin{array}{c}\Pi_{2} \\ \Pi_{3}^{\prime}+\Pi_{3}^{\prime \prime} \Pi_{2}\end{array}\right]$.

Proof: $\quad$ To prove the first claim, let $P_{2}, P_{3} \in \mathbb{R}^{n_{1} \times \mathrm{w}}[\xi]$ be the non strictly proper part of $X_{1} R_{2}^{-1}$ and $X_{1} R_{3}^{-1}$, respectively. The claim follows from the computations $X_{1} \bmod R_{2}=X_{1}-P_{2} R_{2}$; and $\left(X_{1} \bmod R_{3}\right) \bmod R_{2}=\left(X_{1}-P_{3} M R_{2}\right) \bmod R_{2}=X_{1}-$ $P_{3} M R_{2}-\left(P_{2}-P_{3} M\right) R_{2}=X_{1}-P_{2} R_{2}$. The second claim is easily proved by computing $X_{1} \bmod R_{2}$ in terms of $\Pi_{1}$ and $\left(X_{1}\right.$ $\left.\bmod R_{3}\right) \bmod R_{2}$ in terms of $\Pi_{3}$ and $\Pi_{2}$ according to Lemma 3, then factorizing $X_{2}$.

Taking the gluing conditions into account and using Lemma 4, we conclude that when we switch from $\mathfrak{B}_{3}$ to $\mathfrak{B}_{2}$, the condition $Q_{\Psi}(w)\left(t_{i}^{-}\right)-Q_{\Psi}(w)\left(t_{i}^{+}\right) \geq 0$ is equivalent with

$$
Q_{\Psi \bmod R_{3}}(w)\left(t_{i}^{-}\right)-Q_{\left(\Psi \bmod R_{3}\right) \bmod R_{2}}(w)\left(t_{i}^{+}\right) \geq 0
$$

In the following, we aim to express condition (7) in terms of an LMI. In order to do so, consider the factorisation $Q_{\Psi}=$ $X_{1}(d / d t)^{\top} \widetilde{\Psi} X_{1}(d / d t)$ with

$$
\widetilde{\Psi}:=\left[\begin{array}{lll}
\Psi_{11} & \Psi_{12} & \Psi_{13} \\
\Psi_{12}^{\top} & \Psi_{22} & \Psi_{23} \\
\Psi_{13}^{\top} & \Psi_{23}^{\top} & \Psi_{33}
\end{array}\right]
$$

with $\Psi_{11} \in \mathbb{R}^{n_{2} \times n_{2}}, \quad \Psi_{12} \in \mathbb{R}^{n_{2} \times\left(n_{3}-n_{2}\right)}, \quad \Psi_{13} \in \mathbb{R}^{n_{2} \times\left(n_{1}-n_{3}\right)}$, $\Psi_{22} \in \mathbb{R}^{\left(n_{3}-n_{2}\right) \times\left(n_{3}-n_{2}\right)}, \quad \Psi_{23} \in \mathbb{R}^{\left(n_{3}-n_{2}\right) \times\left(n_{1}-n_{3}\right)} \quad$ and $\quad \Psi_{33} \in$ $\mathbb{R}^{\left(n_{1}-n_{3}\right) \times\left(n_{1}-n_{3}\right)}$. From the results of Lemma 2 and Lemma 4 , since the Lyapunov function $Q_{\Psi}$ does not increase when switching from $\mathfrak{B}_{1}$ to $\mathfrak{B}_{2}$, it follows that:

$$
\left[\begin{array}{l}
\Psi_{12}^{\top} \\
\Psi_{13}^{\top}
\end{array}\right]=-\left[\begin{array}{ll}
\Psi_{22} & \Psi_{23} \\
\Psi_{23}^{\top} & \Psi_{33}
\end{array}\right] \Pi_{1}=-\left[\begin{array}{ll}
\Psi_{22} & \Psi_{23} \\
\Psi_{23}^{\top} & \Psi_{33}
\end{array}\right]\left[\begin{array}{c}
\Pi_{2} \\
\Pi_{3}^{\prime}+\Pi_{3}^{\prime \prime} \Pi_{2}
\end{array}\right]
$$

and consequently

$$
\begin{aligned}
& \Psi_{12}^{\top}=-\left(\Psi_{22} \Pi_{2}+\Psi_{23} \Pi_{3}^{\prime}+\Psi_{23} \Pi_{3}^{\prime \prime} \Pi_{2}\right) \\
& \Psi_{13}^{\top}=-\left(\Psi_{23} \Pi_{2}+\Psi_{33} \Pi_{3}^{\prime}+\Psi_{33} \Pi_{3}^{\prime \prime} \Pi_{2}\right) .
\end{aligned}
$$

We now express the entries of the coefficient matrix of $Q_{\Psi \bmod R_{3}}$ in terms of those of $Q_{\Psi \bmod R_{2}}$ as in (8), according to the following lemma.

Lemma 5: Let $Q_{\Psi}=X_{1}(d / d t)^{\top} \widetilde{\Psi} X_{1}(d / d t)$ and $\Pi_{3}:=\left[\Pi_{3}^{\prime} \Pi_{3}^{\prime \prime}\right]$, be as previously defined. Consider the factorisation $Q_{\Psi} \bmod R_{3}=$ $X_{3}(d / d t)^{\top} \widetilde{\widetilde{\Psi}} X_{3}(d / d t)$ with $\widetilde{\widetilde{\Psi}}=\widetilde{\widetilde{\Psi}}^{\top} \in \mathbb{R}^{n_{3} \times n_{3}}$. Consider the partition

$$
\widetilde{\widetilde{\Psi}}:=\left[\begin{array}{ll}
\widetilde{\widetilde{\Psi}}_{11} & \widetilde{\widetilde{\Psi}}_{12} \\
\widetilde{\widetilde{\Psi}}_{12}^{\top} & \widetilde{\widetilde{\Psi}}_{22}
\end{array}\right]
$$

with $\quad \widetilde{\widetilde{\Psi}}_{11} \in \mathbb{R}^{n_{2} \times n_{2}}, \quad \widetilde{\widetilde{\Psi}}_{12} \in \mathbb{R}^{n_{2} \times\left(n_{3}-n_{2}\right)} \quad$ and $\quad \widetilde{\widetilde{\Psi}}_{22} \in$ $\mathbb{R}^{\left(n_{3}-n_{2}\right) \times\left(n_{3}-n_{2}\right)}$. Then $\quad \widetilde{\widetilde{\Psi}}_{11}=\left(\Psi_{11}+\Pi_{3}^{\prime} \Psi_{13}^{\top}+\Psi_{13} \Pi_{3}^{\prime}+\right.$ $\left.\Pi_{3}^{\prime \top} \Psi_{33} \Pi_{3}^{\prime}\right), \quad \widetilde{\widetilde{\Psi}}_{12}=\left(\Psi_{12}+\Pi_{3}^{\prime \top} \Psi_{23}^{\top}+\Psi_{13} \Pi_{3}^{\prime \prime}+\Pi_{3}^{\prime \top} \Psi_{33} \Pi_{3}^{\prime \prime}\right)$ and $\widetilde{\widetilde{\Psi}}_{22}=\left(\Psi_{22}+\Pi_{3}^{\prime \prime \top} \Psi_{23}^{\top}+\Psi_{23} \Pi_{3}^{\prime}+\Pi_{3}^{\prime \prime \top} \Psi_{33} \Pi_{3}^{\prime \prime}\right)$.

Proof: Following the same procedure as in the proof of Lemma 2 and considering the partitions (8) and (11), we conclude that the coefficient matrix (11) can be computed as

$$
\begin{aligned}
{\left[\begin{array}{ll}
\widetilde{\widetilde{\Psi}}_{11} & \widetilde{\widetilde{\Psi}}_{12} \\
\widetilde{\widetilde{\Psi}}_{12}^{\top} & \widetilde{\widetilde{\Psi}}_{22}
\end{array}\right] } & =\left[\begin{array}{cc}
I_{\mathrm{n}\left(\mathfrak{B}_{2}\right)} & 0 \\
0 & I_{\left(n_{3}-n_{2}\right)} \\
\Pi_{3}^{\prime} & \Pi_{3}^{\prime \prime}
\end{array}\right]{ }^{\top} \\
& \times\left[\begin{array}{lll}
\Psi_{11} & \Psi_{12} & \Psi_{13} \\
\Psi_{12}^{\top} & \Psi_{22} & \Psi_{23} \\
\Psi_{13}^{\top} & \Psi_{23}^{\top} & \Psi_{33}
\end{array}\right]\left[\begin{array}{cc}
I_{\mathrm{n}\left(\mathfrak{B}_{2}\right)} & 0 \\
0 & I_{\left(n_{3}-n_{2}\right)} \\
\Pi_{3}^{\prime} & \Pi_{3}^{\prime \prime}
\end{array}\right] .
\end{aligned}
$$

The desired equalities follow by inspection.

Now we return to the proof of Theorem 3 . Note that from inequality (7), we can obtain

$$
\left[\begin{array}{ll}
\widetilde{\widetilde{\Psi}}_{11} & \widetilde{\widetilde{\Psi}}_{12} \\
\widetilde{\widetilde{\Psi}}_{12}^{\top} & \widetilde{\widetilde{\Psi}}_{22}
\end{array}\right]-\left[\begin{array}{cc}
I_{n_{2}} & \Pi_{2}^{\top} \\
0 & 0
\end{array}\right]\left[\begin{array}{ll}
\widetilde{\widetilde{\Psi}}_{11} & \widetilde{\widetilde{\Psi}}_{12} \\
\widetilde{\widetilde{\Psi}}_{12}^{\top} & \widetilde{\widetilde{\Psi}}_{22}
\end{array}\right]\left[\begin{array}{ll}
I_{n_{2}} & 0 \\
\Pi_{2} & 0
\end{array}\right] \geq 0 .
$$

Arguing as in Lemma 2, this inequality holds if and only if $\widetilde{\widetilde{\Psi}}_{12}^{\top}+$ $\widetilde{\widetilde{\Psi}}_{22} \Pi_{2}=0$, or equivalently from Lemma 5 , the condition is satisfied if and only if

$$
\begin{aligned}
\Psi_{12}^{\top}+\Pi_{3}^{\prime \prime \top} \Psi_{13}^{\top}+\Psi_{23} \Pi_{3}^{\prime}+\Pi_{3}^{\prime \prime \top} \Psi_{33} \Pi_{3}^{\prime} \\
\quad=-\left(\Psi_{22}+\Pi_{3}^{\prime \prime \top} \Psi_{23}^{\top}+\Psi_{23} \Pi_{3}^{\prime}+\Pi_{3}^{\prime \prime \top} \Psi_{33} \Pi_{3}^{\prime \prime}\right) \Pi_{2} .
\end{aligned}
$$

Substituting (9) in the latter equation we obtain (10), therefore we conclude that the condition $\widetilde{\widetilde{\Psi}}_{12}^{\top}=-\widetilde{\widetilde{\Psi}}_{22} \Pi_{2}$ is satisfied. Consequently the value of $Q_{\Psi}$ does not increase when switching from $\mathfrak{B}_{3}$ to $\mathfrak{B}_{2}$. It is a matter of straightforward verification to check that when switching from $\mathfrak{B}_{2}$ to $\mathfrak{B}_{3}$ the value of $Q_{\Psi}$ remains the same. This concludes the proof of the theorem.

Proof of Theorem 4: To prove that $M_{\alpha}$, with $0 \leq$ $\alpha \leq 1$, is a strictly positive-real completion define $G_{1}(\xi):=$ $M_{1}(\xi) R_{2}(\xi) R_{1}(\xi)^{-1}$ and $G_{2}(\xi):=M_{2}(\xi) R_{2}(\xi) R_{1}(\xi)^{-1}$. It follows that

$$
\begin{aligned}
&\left(M_{\alpha}(-j \omega) R_{2}(-j \omega) R_{1}(-j \omega)^{-1}\right)^{\top}+M_{\alpha}(j \omega) R_{2}(j \omega) R_{1}(j \omega)^{-1} \\
&=\left(\left(\alpha M_{1}(-j \omega)+(1-\alpha) M_{2}(-j \omega)\right) R_{2}(-j \omega) R_{1}(-j \omega)^{-1}\right)^{\top} \\
&+\left(\alpha M_{1}+(1-\alpha) M_{2}\right) R_{2}(j \omega) R_{1}(j \omega)^{-1} \\
&= \alpha G_{1}(-j \omega)^{\top}+(1-\alpha) G_{2}(-j \omega)^{\top}+\alpha G_{1}(j \omega)+(1-\alpha) G_{2}(j \omega) \\
&= \alpha\left(G_{1}(-j \omega)^{\top}+G_{1}(j \omega)\right)+(1-\alpha)\left(G_{2}(-j \omega)^{\top}+G_{2}(j \omega)\right) .
\end{aligned}
$$

The claim follows from the fact that $G_{1}$ and $G_{2}$ are strictly positivereal. We now prove that $\Sigma^{\prime}$ is asymptotically stable. Let $\widetilde{\Psi}_{i}:=$ $\left[\begin{array}{cc}\Psi_{i, 11} & -\Pi^{\top} \Psi_{i, 22} \\ -\Psi_{i, 22} \Pi & \Psi_{i, 22}\end{array}\right]>0, i=1,2$ be as in Theorem, corresponding to the solution of (4) using the positive-real completion $M_{i}$, $i=1,2$, respectively. Following straightforward computations, it can be proven that:

$$
\begin{aligned}
\Psi_{\alpha}(\zeta, \eta) & :=\alpha \Psi_{1}(\zeta, \eta)+(1-\alpha) \Psi_{2}(\zeta, \eta) \\
& =\frac{R_{1}(\zeta)^{\top} M_{\alpha}(\eta) R_{2}(\eta)+R_{2}(\zeta)^{\top} M_{\alpha}(\zeta)^{\top} R_{1}(\eta)-Q_{\alpha}(\zeta)^{\top} Q_{\alpha}(\eta)}{\zeta+\eta}
\end{aligned}
$$

for some $Q_{\alpha} \in \mathbb{R}^{\bullet \times w}[\xi]$. It follows that the convex combination of the two-variable polynomial matrices $\Psi_{1}$ and $\Psi_{2}$ yields the coefficient matrix $\widetilde{\Psi}_{\alpha}:=\left[\begin{array}{cc}\Psi_{\alpha, 11} & -\Pi^{\top} \Psi_{\alpha, 22} \\ -\Psi_{\alpha, 22} \Pi & \Psi_{\alpha, 22}\end{array}\right]>0$, as in Theorem, and hence $Q_{\Psi_{\alpha}}$ is a Lyapunov function for $\Sigma$. Finally, to conclude the proof, apply Theorem 3. 


\section{REFERENCES}

[1] W. A. Coppel, Linear Systems, Notes in Pure Mathematics. Canberra, Act., Australia: Australian Nat. Univ., 1972

[2] J. P. Hespanha and A. S. Morse, "Switching between stabilizing controllers," Automatica, vol. 38, no. 11, pp. 1905-1917, 2002.

[3] T. Kailath, Linear Systems. Upper Saddle River, NJ, USA: PrenticeHall Information and System Sciences Series, 1980.

[4] T. Kailath and A. H. Sayed, Fast Reliable Algorithms for Matrices With Structure. Philadelphia, PA, USA: SIAM, 1999.

[5] A. G. Kelkar and S. M. Joshi, "Robust control of non-passive systems via passification," in Proc. American Control Conf., Jun. 1997, pp. 2657-2661.

[6] D. Liberzon, Switching in Systems and Control. Boston, MA, USA: Birkhauser, 2003.

[7] O. Mason and R. Shorten, "On linear copositive Lyapunov functions and the stability of switched positive linear systems," IEEE Trans. Autom. Control, vol. 52, no. 7, pp. 1346-1349, Jul. 2007.

[8] J. C. Mayo-Maldonado and P. Rapisarda, "On positive-realness and stability of switched linear differential systems," in Proc. 52nd IEEE Annu. Conf. Decision and Control (CDC), Dec. 2013, pp. 162-167.

[9] J. C. Mayo-Maldonado and P. Rapisarda, "Modelling of switching dynamics in electrical systems," in Proc. MTNS, 2014, pp. 985-992.

[10] J. C. Mayo-Maldonado, P. Rapisarda, and P. Rocha, "Stability of switched linear differential systems," IEEE Trans. Autom. Control, vol. 59, no. 8, pp. 2038-2051, Aug. 2014.

[11] P. Youbin, D. Vrancic, and R. Hanus, "Anti-windup, bumpless, conditioned transfer techniques for PID controllers," IEEE Cont. Syst., vol. 16, no. 4, pp. 48-57, Aug. 1996.
[12] P. Rocha, J. C. Willems, P. Rapisarda, and D. Napp, "On the stability of switched behavioral systems," in Proc. 50th IEEE Conf. Decision and Control and European Control Conference (CDC-ECC), 2011, pp. $1534-1538$.

[13] S. Sajja, M. Corless, E. Zeheb, and R. Shorten, "Comments and observations on the passivity of descriptor systems in state space," Int. J. Control, vol. 86, no. 1, pp. 120-126, 2013.

[14] S. Sajja, M. Corless, E. Zeheb, and R. Shorten, "On dimensionality reduction and the stability of a class of switched descriptor systems," Automatica, vol. 49, no. 6, pp. 1855-1860, 2013.

[15] R. Shorten, M. Corless, K. Wulff, S. Klinge, and R. Middleton, "Quadratic stability and singular SISO switching systems," IEEE Trans. Autom. Control, vol. 54, no. 11, pp. 2714-2718, Nov. 2009.

[16] R. Shorten, K. S. Narendra, and O. Mason, "A result on common quadratic Lyapunov functions," IEEE Trans. Autom. Control, vol. 48, no. 1, pp. 110-113, Jan. 2003.

[17] R. N. Shorten and K. S. Narendra, "On common quadratic Lyapunov functions for pairs of stable LTI systems whose system matrices are in companion form," IEEE Trans. Autom. Control, vol. 48, no. 4, pp. 618-621, Apr. 2003.

[18] S. Trenn, "Distributional Differential Algebraic Equations," Ph.D. dissertation, Technische Universität Ilmenau, Thuringia, Germany, 2009.

[19] J. C. Willems and H. L. Trentelman, "On quadratic differential forms," SIAM J. Control Optimiz., vol. 36, pp. 1703-1749, 1998.

[20] E. Zeheb, R. Shorten, and S. S. K. Sajja, "Strict positive realness of descriptor systems in state space," Int. J. Control, vol. 83, no. 9, pp. 17991809,2010 